8th Alexander Friedmann International Seminar

on Gravitation and Cosmology

International Journal of Modern Physics: Conference Series

Vol. 3 (2011) 150-160

(C) World Scientific Publishing Company

DOI: $10.1142 / \mathrm{S} 2010194511001243$

\title{
TORSION CORRECTIONS IN BRANE WORLD GRAVITY
}

\author{
R. MAIER \\ ICRA department, Centro Brasileiro de Pesquisas Fúsicas, \\ Rua Dr. Xavier Sigaud, 150, Urca, Rio de Janeiro, 22290-180, Brazil \\ rodmaier@cbpf.br
}

Received 7 June 2011

\begin{abstract}
In the frame of brane world theory the effects of torsion fields are examined. Considering a five dimensional Non-Riemannian bulk with a noncompact extra dimension, we derive the modified Einstein field equations in a four dimensional (3-brane) arbitrary manifold embedded in this bulk. The necessary matching conditions are investigated assuming that the torsion in the bulk is continuous. In this context the extrinsic curvature is connected to the matter content restricted to the brane and the torsion components of the bulk. As a final result we observe that the corrections - due to torsion fields - in the modified field equations depend crucially on the embedding that is taken. Therefore, by considering a simple embedding, we develop a cosmological model that describes a flat FLRW embedded in a 5-dimensional de Sitter (or Anti de Sitter) spacetime, where a 5-dimensional cosmological constant emerges from the torsion components of the bulk.
\end{abstract}

Keywords: Braneworld theory; torsion; cosmology.

PACS numbers: 11.25.Hf, $123.1 \mathrm{~K}$

\section{Introduction}

Brane world theory, ${ }^{1-5}$ has been an interesting option within extra dimension theories over the last years. In this scenario, extra dimensions are introduced by a bulk space, and all the matter content of the Universe would be trapped on a brane with three spatial dimensions. Only gravitons would be allowed to leave the surface and move in the full bulk. ${ }^{2}$ At low energies general relativity is recovered but at high energies significant changes are introduced in the gravitational dynamics. Among several solutions provided by this scenario, we can mention the answers given to the hierarchy problem, ${ }^{3}$ the avoidance of the initial singularity of the Universe,${ }^{4-5}$ and inflationary or late time accelerating models. ${ }^{6-8}$

In this paper, we propose to include torsion effects in the brane world scenario. Even though, up to date, there is no experimental evidence for introducing torsion in gravity, there are some theoretical arguments in favor to consider torsion fields as a desired component in any spacetime theory. ${ }^{9-11}$ The Cauchy problem in gravitational collapse, ${ }^{12}$ for instance, is intrinsicaly related to the affine structure 
of the manifold. Thus, one might hope to avoid its divergences while including torsion effects. It has been shown, ${ }^{13}$ that brane world corrections to the Schwarzschild metric tend to attenuate gravitational lensing effects and the advance of planetary perihelia. Although these corrections are not relevant when considering the experimental tests of General Relativity, these might be important for masses and/or scales much larger than that of the solar system. Therefore, torsion can play an important role in these matters.

In this first analysis, we shall consider the torsion as a fundamental tensor completely independent from the metric that defines the affine structure of the bulk. Therefore, for a five dimensional bulk, there is no a priori constraint in the 50 components of its torsion field.

In this paper we firstly set the conventions in order to introduce torsion components in the embedding Gauss-Codazzi equations. Assuming a five dimensional Einstein's equation with torsion, we derive the modified field equations on the brane by considering the junction conditions with the $Z_{2}$ symmetry. Finally, we present a simple solution for the embedding of a flat four dimensional FLRW spacetime in a five dimensional de Sitter space where the field equations need not to be feeded with a cosmological constant. Last section is reserved for comments and final remarks.

\section{Conventions And Basic Equations}

Let us consider a $N$-dimensional Non-Riemannian space endowed with a metric tensor ${ }^{(N)} g_{a b}$ and a non-trivial affine structure due to torsion terms ${ }^{(N)} T_{. b c}^{a}$. The connection can be defined as

$$
{ }^{(N)} \Gamma_{b c}^{a}={ }^{(N)}\left\{\begin{array}{l}
a \\
b c
\end{array}\right\}+{ }^{(N)} K^{a} \cdot b c,
$$

where ${ }^{(N)}\left\{\begin{array}{c}a \\ b c\end{array}\right\}$ is the Christoffel symbol and ${ }^{(N)} K_{. b c}^{a}$ is the contortion tensor. Thus, the covariant derivative of an arbitrary vector field $Z_{a}$ is defined as

$$
\nabla_{b} Z_{a} \equiv Z_{a, b}-{ }^{(N)} \Gamma_{b a}^{c} Z_{c}
$$

The torsion

$$
{ }^{(N)} T_{. b c}^{a} \equiv{ }^{(N)} \Gamma_{b c}^{a}-{ }^{(N)} \Gamma_{c b}^{a}
$$

together with the metricity condition ${ }^{(N)} \nabla_{c} g_{a b}=0$ allow us to write the contortion as

$$
{ }^{(N)} K_{a b c}=\frac{1}{2}\left({ }^{(N)} T_{a b c}+{ }^{(N)} T_{b a c}+{ }^{(N)} T_{c a b}\right),
$$

which has the anti-symmetry ${ }^{(N)} K_{a b c}=-{ }^{(N)} K_{c b a}$. The curvature tensor ${ }^{(N)} R_{a b c}^{d}$ is then defined by

$$
\nabla_{c} \nabla_{b} Z_{a}-\nabla_{b} \nabla_{c} Z_{a}={ }^{(N)} R_{a b c}^{d} Z_{d}+T_{. b c}^{d} \nabla_{d} Z_{a},
$$

and can be separeted in its Riemannian and non-Riemannian parts as

$$
{ }^{(N)} R_{b c d}^{a}={ }^{(N)} \tilde{R}_{b c d}^{a}+{ }^{(N)} K_{b c d}^{a} .
$$


${ }^{(N)} \tilde{R}_{b c d}^{a}$ is the Riemanian tensor defined only with the Christoffels, ${ }^{14-15}$ and

$$
\begin{aligned}
{ }^{(N)} K_{b c d}^{a}={ }^{(N)} D_{c}{ }^{(N)} K^{a} \cdot d b-{ }^{(N)} D_{d}{ }^{(N)} K^{a} \cdot c b \\
\\
+{ }^{(N)} K^{m} \cdot d b{ }^{(N)} K^{a} \cdot c m-{ }^{(N)} K^{m} \cdot c b
\end{aligned}
$$

where ${ }^{(N)} D$ means covariant derivative constructed only with the Christoffel symbols.

In our study, we consider a bulk manifold $U_{5}$ with coordinates $\left\{Y^{A}, A=0, . ., 4\right\}$ and the brane $V_{4}$ as a subspace of $U_{5}$ with coordinates $\left\{x^{\alpha}, \alpha=0, . ., 3\right\}$. Therefore, there must exist a function set

$$
Y^{A}=f^{A}\left(x^{0}, \ldots, x^{n}\right), \quad A=0, . ., 4
$$

such that

$$
{ }^{(4)} g_{\alpha \beta}={ }^{(5)} g_{A B} Y_{, \alpha}^{A} Y_{, \beta}^{B},
$$

where ${ }^{(5)} g_{A B}$ is the metric tensor in $U_{5}$ and ${ }^{(4)} g_{\alpha \beta}$ is the so-called induced geometry in $V_{4}$. Equations (7) stand as the parametric equations of $V_{4}$ as a subspace of $U_{5}$.

Let $X^{A}$ be a unitary vector field in $U_{5}$ orthogonal to $V_{4}$. That is,

$$
\text { (5) } g_{A B} Y_{\alpha}^{A} X^{B}=0
$$

and

$$
{ }^{(5)} g_{A B} X^{A} X^{B}=\epsilon= \pm 1
$$

In our convention $\epsilon=+1$ means a timelike extra dimension. For $\epsilon=-1$ we have a spacelike extra dimension.

Furthermore of $V_{4}$ and $U_{5}$ being metric spaces, throughout this paper we will assume that the torsion components do not vanish, in general, in any of these two manifolds.

\section{Field Equations}

In order to obtain the modified gravitational field equations on the brane, let us assume that the bulk field equations read

$$
{ }^{(5)} G_{A B}-\Lambda_{5}{ }^{(5)} g_{A B} \equiv{ }^{(5)} \tilde{G}_{A B}+{ }^{(5)} L_{A B}-\Lambda_{5}{ }^{(5)} g_{A B}=\kappa_{5}^{2(5)} T_{A B} \quad,
$$

where

$$
\begin{aligned}
& { }^{(5)} \tilde{G}_{A B} \equiv{ }^{(5)} \tilde{R}_{A B}-\frac{1}{2}{ }^{(5)} \tilde{R}^{(5)} g_{A B}, \\
& { }^{(5)} L_{A B} \equiv{ }^{(5)} K_{A B}-\frac{1}{2}{ }^{(5)} K^{(5)} g_{A B},
\end{aligned}
$$

with ${ }^{(5)} K_{A B} \equiv{ }^{(5)} K_{A C B}^{C}$ and ${ }^{(5)} K \equiv{ }^{(5)} g^{A B}{ }^{(5)} K_{A B}$.

Defining the extrinsic curvature of $V_{4}$ in $U_{5}$ as

$$
\Omega_{\alpha \beta}=-{ }^{(5)} g_{A B} Y_{, \alpha}^{A} Y_{, \beta}^{C}{ }^{(5)} \nabla_{C} X^{B},
$$


where ${ }^{(5)} \nabla$ is the covariant derivative built with the connection (1), it is straightforward to show, ${ }^{15}$

$$
{ }^{(4)} R_{\alpha \beta \gamma \delta}={ }^{(5)} R_{A B C D} Y_{, \alpha}^{A} Y_{, \beta}^{B} Y_{, \gamma}^{C} Y_{, \delta}^{D}+\epsilon\left(\Omega_{\beta \delta} \Omega_{\alpha \gamma}-\Omega_{\beta \gamma} \Omega_{\alpha \delta}\right),
$$

and

$$
{ }^{(4)} \nabla_{\gamma} \Omega_{\alpha \beta}-{ }^{(4)} \nabla_{\beta} \Omega_{\alpha \gamma}={ }^{(5)} R_{A B C D} X^{A} Y_{, \alpha}^{B} Y_{, \gamma}^{C} Y_{, \beta}^{D}-T_{, \gamma \beta}^{\sigma} \Omega_{\alpha \sigma} .
$$

Equations (15) and (16) are the Gauss-Codazzi equations for nonvanishing torsion components. Contracting $\beta$ and $\delta$ in the Gauss equation we get

$$
\begin{array}{r}
{ }^{(4)} G_{\alpha \gamma}={ }^{(5)} R_{A C} Y_{, \alpha}^{A} Y_{, \gamma}^{C}-\epsilon{ }^{(5)} R_{A B C D} Y_{, \alpha}^{A} X^{B} Y_{, \gamma}^{C} X^{D} \\
+\epsilon\left(\Omega_{\alpha \gamma} \Omega-\Omega_{\alpha \delta} \Omega_{\gamma}^{\delta}\right)-\frac{1}{2}{ }^{(4)} g_{\alpha \gamma}{ }^{(4)} R
\end{array}
$$

with ${ }^{(4)} G_{\alpha \beta} \equiv{ }^{(4)} \tilde{G}_{\alpha \beta}+L_{\alpha \beta}$, and with an another contraction

$$
{ }^{(4)} R={ }^{(5)} R-2 \epsilon^{(5)} R_{A C} X^{A} X^{C}+\epsilon\left(\Omega^{2}-\Omega_{\gamma \delta} \Omega^{\delta \gamma}\right) \text {. }
$$

Using this result in (17) we end up with

$$
\begin{aligned}
{ }^{(4)} G_{\alpha \gamma}= & { }^{(5)} G_{A C} Y_{, \alpha}^{A} Y_{, \gamma}^{C}-\epsilon^{(5)} R_{A B C D} Y_{, \alpha}^{A} X^{B} Y_{, \gamma}^{C} X^{D} \\
& +\epsilon\left(\Omega_{\alpha \gamma} \Omega-\Omega_{\alpha \delta} \Omega_{\gamma}^{\delta}\right)-\frac{1}{2} \epsilon{ }^{(4)} g_{\alpha \gamma}\left(\Omega^{2}-\Omega_{\beta \delta} \Omega^{\delta \beta}\right) \\
& +\epsilon^{(5)} R_{A C} X^{A} X^{C(4)} g_{\alpha \gamma} .
\end{aligned}
$$

Since Einstein's equation determines only the trace part of the curvature tensor, it is usefull to decompose it in terms of its traces and the Weyl tensor, the trace-free part,

$$
\begin{gathered}
{ }^{(5)} \tilde{R}_{A B C D}={ }^{(5)} C_{A B C D}+\frac{2}{3}\left[{ }^{(5)} g_{A[C}{ }^{(5)} \tilde{R}_{D] B}-{ }^{(5)} g_{B[C}{ }^{(5)} \tilde{R}_{D] A}\right] \\
-\frac{1}{6}{ }^{(5)} \tilde{R}^{(5)} g_{A[C}{ }^{(5)} g_{D] B} \quad
\end{gathered}
$$

where $Q_{[A B]} \equiv \frac{1}{2}\left(Q_{A B}-Q_{B A}\right)$ for an arbitrary tensor $Q_{A B}$. Using this decomposition, equation (19) can now be written as

$$
\begin{aligned}
{ }^{(4)} G_{\alpha \gamma} & =\left(\frac{2}{3}{ }^{(5)} \tilde{R}_{A C}+{ }^{(5)} K_{A C}\right) Y_{, \alpha}^{A} Y_{, \gamma}^{C}+\epsilon\left(\Omega_{\alpha \gamma} \Omega-\Omega_{\alpha \delta} \Omega_{\gamma}^{\delta}\right) \\
& -\frac{1}{2} \epsilon\left(\Omega^{2}-\Omega_{\beta \delta} \Omega^{\delta \beta}\right)^{(4)} g_{\alpha \gamma}-\epsilon E_{\alpha \gamma}-\epsilon J_{\alpha \gamma} \\
& +\left[\epsilon\left(\frac{2}{3}^{(5)} \tilde{R}_{A C}+{ }^{(5)} K_{A C}\right) X^{A} X^{C}-\frac{5}{12}^{(5)} \tilde{R}-\frac{1}{2}{ }^{(5)} K\right]{ }^{(4)} g_{\alpha \gamma},
\end{aligned}
$$

where we have defined

$$
\begin{aligned}
E_{\alpha \gamma} & \equiv{ }^{(5)} C_{A B C D} Y_{, \alpha}^{A} X^{B} Y_{, \gamma}^{C} X^{D}, \\
J_{\alpha \gamma} & \equiv{ }^{(5)} K_{A B C D} Y_{, \alpha}^{A} X^{B} Y_{, \gamma}^{C} X^{D},
\end{aligned}
$$


Using the bulk field equations (11), we can rewrite equation (20) as

$$
\begin{aligned}
{ }^{(4)} G_{\alpha \gamma} & =\frac{2}{3} \kappa_{5}^{2}\left[{ }^{(5)} T_{A C} Y_{, \alpha}^{A} Y_{, \gamma}^{C}+\left(\epsilon^{(5)} T_{A C} X^{A} X^{C}-\frac{1}{4}{ }^{(5)} T\right){ }^{(4)} g_{\alpha \gamma}\right]+\frac{1}{2} \Lambda_{5}{ }^{(4)} g_{\alpha \gamma} \\
& +\epsilon\left(\Omega_{\alpha \gamma} \Omega-\Omega_{\alpha \delta} \Omega_{\gamma}^{\delta}\right)-\frac{1}{2} \epsilon^{(4)} g_{\alpha \gamma}\left(\Omega^{2}-\Omega_{\beta \delta} \Omega^{\delta \beta}\right)-\epsilon\left(E_{\alpha \gamma}+J_{\alpha \gamma}\right) \\
& +\frac{1}{3}{ }^{(5)} K_{A C} Y_{, \alpha}^{A} Y_{, \gamma}^{C}+\frac{1}{3}\left(\epsilon^{(5)} K_{A C} X^{A} X^{C}-\frac{1}{4}{ }^{(5)} K\right){ }^{(4)} g_{\alpha \gamma} .
\end{aligned}
$$

These are the modified Einstein's field equation in the brane when one considers nonvanishing torsion for the bulk. The torsion manifests itself not only introducing extra terms in the field equations, but also inducing a torsion tensor on the brane.

To describe the evolution of the gravitational field restricted to the brane we still have to specify how the brane is curved with respect to the bulk. In the next section we will analise the necessary junction conditions (using the $Z_{2}$ symmetry) in order to connect the extrinsic curvature to the matter distribution restricted to the brane.

\section{Junction Conditions}

From General Relativity we know that the Israel junction conditions must be satisfied in order to connect the discontinuities of the matter distribution with the geometry of the spacetime. ${ }^{16}$ As in brane world scenarios we assume that the matter distribution is restricted to a 4-dimensional brane $(1+3)$ embedded in a 5 -dimensional bulk, there are analogous consistency conditions relating discontinuities of the energy-momentum tensor across the brane with the extrinsic curvature. In this section we will examine this issue and see how this discontinuity can be related to the extrinsic curvature of the brane.

From now on we are going to assume that the metric is continuous in the bulk. Therefore, any discontinuity of its first derivative across the brane will be perpendicular to the brane. That is

$$
\left[{ }^{(5)} g_{A B}\right]_{V_{4}}=0,\left[{ }^{(5)} g_{A B, C}\right]_{V_{4}}=\chi_{A B} X_{C} \quad,
$$

where $[f]_{V_{4}}$ means the discontinuity of an arbitrary function $f$ across $V_{4},{ }^{17-19}$ and $\chi_{A B}=\chi_{B A}$.

Using Einstein's equations we will be able to connect this discontinuity with the matter singular behavior across the brane. Nevertheless, we still have to specify how the torsion field changes due to the matter discontinuity. From the field equation we get that the torsion first derivative should be discontinuous if we consider matter discontinuities. Therefore, it is reasonable to assume that the torsion tensor (or equivalently the contortion) is continuous just as the metric tensor, but its first derivative is discontinuous.

Let us consider a gaussian coordinate system given by

$$
d s^{2}=\epsilon d y^{2}+{ }^{(4)} g_{\alpha \beta}\left(x^{\gamma}\right) d x^{\alpha} d x^{\beta},
$$


where $y$ denotes the extra dimension and $X^{A} \equiv \delta_{y}^{A}$. Contracting $\alpha$ and $\gamma$ in (15) we get

$$
{ }^{(4)} R_{\beta \delta}={ }^{(5)} R_{A B} Y_{, \beta}^{A} Y_{, \delta}^{B}-\epsilon^{(5)} R_{y B y D} Y_{, \beta}^{B} Y_{, \delta}^{D}+\epsilon\left(\Omega_{\beta \delta} \Omega-\Omega_{\beta \gamma} \Omega_{\delta}^{\gamma}\right) .
$$

Using the above coordinate system one can easily show that

$$
\begin{aligned}
& { }^{(5)} R_{y B y D} Y_{, \alpha}^{B} Y_{, \beta}^{D}=\Omega_{\alpha \beta, y}-{ }^{(5)} K_{y y B, D} Y_{, \alpha}^{B} Y_{, \beta}^{D} \\
& +\left[\frac{1}{4}{ }^{(5)} g^{C E}{ }^{(5)} g_{C D, y}{ }^{(5)} g_{B E, y}-\frac{1}{2}{ }^{(5)} g^{E F(5)} g_{B F, y} K_{y D E}+\tilde{\Gamma}_{D B}^{E} K_{y y E}\right. \\
& \left.+\frac{1}{2}{ }^{(5)} g^{E F^{(5)}} g_{D F, y} K_{E y B}+K_{. D B}^{M} K_{y y M}-K_{. y B}^{M} K_{y D M}\right] Y_{, \alpha}^{B} Y_{, \beta}^{D} .
\end{aligned}
$$

Now Eq. (23) may be rewritten as

$$
{ }^{(5)} R_{A B} Y_{, \alpha}^{A} Y_{, \beta}^{B} \equiv{ }^{(5)} R_{\alpha \beta}=\epsilon \Omega_{\alpha \beta, y}+Z_{\alpha \beta} \quad,
$$

where $Z_{\alpha \beta}$ stands for continuous and bounded terms in a finite region that circumscribe the brane.

Considering the singular behavior of matter distribution across the brane, the 5 -dimensional general energy-momentum tensor is written as

$$
{ }^{(5)} T_{A B}={ }^{(5)} \tilde{T}_{A B}+{ }^{(5)} \mathcal{T}_{A B} \delta(y),
$$

where ${ }^{(5)} \tilde{T}_{A B}$ and ${ }^{(5)} \mathcal{T}_{A B}$ denote respectively the continuous and discontinuous components of ${ }^{(5)} T_{A B}$ across the brane. Moreover, we are going to assume ${ }^{(5)} \mathcal{T}_{A B} X^{A}=0$ and

$$
\mathcal{T}_{\alpha \beta} \equiv{ }^{(5)} \mathcal{T}_{A B} Y_{, \alpha}^{A} Y_{, \beta}^{B}=\tau_{\alpha \beta}-\sigma^{(4)} g_{\alpha \beta},
$$

where $\tau_{\alpha \beta}$ denotes the matter content restricted to the brane and $\sigma$ is defined as the brane tension.

From the bulk field equations (11) we get

$$
{ }^{(5)} R_{A B}=\kappa_{5}^{2}\left[\mathcal{T}_{A B}-\frac{1}{3} \mathcal{T}^{(5)} g_{A B}\right] \delta(y)+\kappa_{5}^{2}\left[\tilde{T}_{A B}-\frac{1}{3} \tilde{T}^{(5)} g_{A B}\right]-\frac{2}{3} \Lambda_{5}{ }^{(5)} g_{A B}
$$

According to our initial assumption, $Z_{\alpha \beta},{ }^{(5)} \tilde{T}_{A B}$ and ${ }^{(4)} g_{\alpha \beta}$ are bounded functions around $y=0$. Therefore, using equations (24)-(27), we have

$$
\epsilon \lim _{\xi \rightarrow 0} \int_{-\xi}^{+\xi} \Omega_{\alpha \beta, y} d y=\lim _{\xi \rightarrow 0} \int_{-\xi}^{+\xi}{ }^{(5)} R_{\alpha \beta} d y=\kappa_{5}^{2}\left[\mathcal{T}_{\alpha \beta}-\frac{1}{3} \mathcal{T}^{(5)} g_{\alpha \beta}\right] .
$$

Imposing the so-called $Z_{2}$ symmetry, ${ }^{1}$ and remembering that $\mathcal{T}_{\alpha \beta}$ is a symmetric tensor, the symmetrical part of the extrinsic curvature is given by

$$
\Omega_{(\alpha \beta)}=\frac{1}{2} \epsilon \kappa_{5}^{2}\left[\mathcal{T}_{\alpha \beta}-\frac{1}{3} \mathcal{T}^{(5)} g_{\alpha \beta}\right] .
$$

The anti-symmetrical part of the extrinsic curvature can easily be obtained by using definitions (2) and (14). Thus, the general form of the extrinsic curvature can be written as

$$
\Omega_{\alpha \beta}=\frac{1}{2} \epsilon \kappa_{5}^{2}\left[\tau_{\alpha \beta}-\frac{1}{3}(\tau-\sigma) g_{\alpha \beta}\right]+\frac{1}{2} T_{A B C} X^{A} Y_{, \alpha}^{B} Y_{, \beta}^{C} .
$$


Substituting all these terms in Eq. (21), we end up with the following modified Einstein's field equation on the brane

$$
\begin{gathered}
{ }^{(4)} G_{\alpha \beta}+\Lambda_{4}{ }^{(4)} g_{\alpha \beta}=8 \pi G_{N} \tau_{\alpha \beta}+\epsilon \kappa_{5}^{4} \Pi_{\alpha \beta}+\epsilon F_{\alpha \beta}-\epsilon E_{\alpha \beta}-\epsilon J_{\alpha \beta} \\
+\frac{1}{3}{ }^{(5)} L_{A B} Y_{, \alpha}^{A} Y_{, \beta}^{B}+\frac{1}{3}\left(\epsilon^{(5)} L_{A B} X^{A} X^{B}-\frac{1}{4}{ }^{(5)} L\right){ }^{(4)} g_{\alpha \beta},
\end{gathered}
$$

where we have defined

$$
\begin{aligned}
\Lambda_{4} \equiv & -\frac{1}{2} \Lambda_{5}+\frac{\epsilon}{12} \kappa_{5}^{4} \sigma^{2}, \quad G_{N} \equiv \kappa_{5}^{4} \frac{\epsilon \sigma}{48 \pi} \\
F_{\alpha \beta} \equiv & \frac{2}{3} \kappa_{5}^{2}\left[\epsilon^{(5)} T_{A B} Y_{\alpha}^{A} Y_{, \beta}^{B}+\left({ }^{(5)} T_{A B} X^{A} X^{B}-\frac{1}{4} \epsilon^{(5)} T\right){ }^{(4)} g_{\alpha \beta}\right] \\
\Pi_{\alpha \beta} \equiv & -\frac{1}{4}\left(\tau_{\alpha \gamma}+\frac{\epsilon}{\kappa_{5}^{2}} T_{A \alpha \gamma} X^{A}\right)\left(\tau_{\beta}^{\gamma}+\frac{\epsilon}{\kappa_{5}^{2}} T_{B}{ }_{\beta} X^{B}\right) \\
& +\frac{1}{8}\left(\tau_{\delta \gamma} \tau^{\gamma \delta}+\frac{1}{\kappa_{5}^{4}} T_{A \delta \gamma} T_{B}{ }^{\gamma \delta} X^{A} X^{B}\right){ }^{(4)} g_{\alpha \beta} \\
& +\frac{\tau}{12}\left(\tau_{\alpha \beta}+\frac{\epsilon}{\kappa_{5}^{2}} T_{A \alpha \beta} X^{A}\right)-\frac{1}{24} \tau^{2(4)} g_{\alpha \beta}+\frac{\epsilon \sigma}{6 \kappa_{5}^{2}} T_{A \alpha \beta} X^{A}
\end{aligned}
$$

As the Newton's constant $G_{N}$ has to be positive, the brane tension $\sigma$ has to be a positive value for a timelike extra dimension (or a negative value for a spacelike extra dimension). Furthermore, if one sets $\Lambda_{5}=0$, the sign of the effective four dimensional cosmological constant $\Lambda_{4}$ is also fixed by the nature of the extra dimension. ${ }^{20}$ The tensor $F_{\alpha \beta}$ represents the contribution of the 5-dimensional energy-momentum tensor and $\Pi_{\alpha \beta}$ are correction terms quadratic in $\tau_{\alpha \beta}$ that are no longer symmetric due to presence of the torsion terms $T_{A \alpha \beta} X^{A}$. One should also note that ${ }^{(4)} G_{\alpha \beta}$ in (31) include torsion terms. Hence, in general, ${ }^{(4)} G_{\alpha \beta}$ is also not a symmetric tensor.

We have consistently introduced torsion effects in a 5-dimensional bulk and derived the modified 4-dimensional field equation in the brane world scenario. To complete our analysis, we propose a specific example that allow us to construct a model where the brane is described by a flat FLRW metric.

\section{A Simple Solution}

In this section we are going to construct a solution of the field equation (11) that admits a four dimensional flat FLRW metric as subspace. Bearing the usual brane world scenarios in mind, we shall assume a five dimensional geometry that describes a vacuum configuration in the bulk. In the coordinate system $(u, v, \chi, \vartheta, \psi)$ the metric can be written as

$$
\mathrm{d} s^{2}=H_{\Lambda}^{2} v^{2} \mathrm{~d} u^{2}+\frac{\epsilon}{H_{\Lambda}^{2} v^{2}} \mathrm{~d} v^{2}-v^{2}\left[\mathrm{~d} \chi^{2}+\chi^{2} \mathrm{~d} \Omega^{2}\right]
$$

where $d \Omega=\mathrm{d} \vartheta^{2}+\sin ^{2} \vartheta \mathrm{d} \psi^{2}$ is the solid angle and $H_{\Lambda}^{2}$ is an arbitrary constant. Therefore the Ricci scalar reads

${ }^{(5)} R=-20 \epsilon H_{\Lambda}^{2}$ 
That is, for a timelike extra dimension we have a de Sitter bulk. On the contrary, for a spacelike extra dimension we have an Anti de Sitter spacetime.

In order to find a simple solution of the five dimensional field equations (11) we shall propose the following ansatz for the torsion in the bulk

$$
\left.\left.T_{A B C}=\alpha^{(5)} g_{A[B} \varphi, C\right] \quad \Rightarrow \quad K_{A B C}=\alpha^{(5)} g_{B[A} \varphi, C\right]
$$

where $\alpha$ is an arbitrary constant and $\varphi$ is a 5 -dimensional scalar field.

Using the above metric and assuming that $\varphi=\varphi(v)$ with

$$
\frac{\mathrm{d} \varphi}{\mathrm{d} v}=-\frac{1}{\alpha v}
$$

straightforward but long calculation shows that

$$
\begin{aligned}
{ }^{(5)} \tilde{G}_{A B} & =6 \epsilon H_{\Lambda}^{2(5)} g_{A B} \\
{ }^{(5)} L_{A B} & \equiv{ }^{(5)} K_{A B}-\frac{1}{2}{ }^{(5)} K^{(5)} g_{A B}=-6 \epsilon H_{\Lambda}^{2(5)} g_{A B} .
\end{aligned}
$$

Therefore, our ansatz satisfies the field equation (11) as long as we take $\Lambda_{5}=0$. Once we have a 5-dimensional de Sitter or Anti de Sitter solution, one can verify that the flat 4-dimensional FLRW spacetime

$$
\mathrm{d} s^{2}=\mathrm{d} t^{2}-a^{2}(t)\left[\mathrm{d} r^{2}+r^{2}\left(\mathrm{~d} \theta^{2}+\sin ^{2} \theta \mathrm{d} \phi^{2}\right)\right]
$$

can be embedded in the spacetime (32) through the following embedding functions

$$
\begin{aligned}
Y^{0} & =\frac{1}{H_{\Lambda}} \int \frac{\mathrm{d} t}{a} \sqrt{1-\frac{\epsilon \dot{a}^{2}}{H_{\Lambda}^{2} a^{2}}} \quad, \quad Y^{1}=v(t)=-a(t) \\
Y^{2} & =\chi=r \quad, \quad Y^{3}=\vartheta=\theta \quad, \quad Y^{4}=\psi=\phi .
\end{aligned}
$$

In this coordinate system the normal vectors are given by

$$
X^{A}= \pm\left(\frac{\epsilon \dot{a}}{H_{\Lambda}^{2} a^{2}}, \sqrt{H_{\Lambda}^{2} a^{2}-\epsilon \dot{a}^{2}}, 0,0,0\right) \quad .
$$

Considering that the matter content restricted to the brane is described by a perfect fluid

$$
\tau_{\alpha \beta}=(\rho+p) V_{\alpha} V_{\beta}-p^{(4)} g_{\alpha \beta} \quad,
$$

one can show that the corrected field equations in the brane read

$$
\begin{aligned}
\left(\frac{\dot{a}}{a}\right)^{2} & =\frac{2 \pi G_{N}}{3} \rho\left[1-\frac{\epsilon}{2|\sigma|} \rho\right]-\frac{\Lambda_{4}}{12}, \\
\frac{\ddot{a}}{a} & =-\frac{2 \pi G_{N}}{3}\left[\rho+3 p-\frac{\epsilon}{|\sigma|} \rho(2 \rho+3 p)\right]-\frac{1}{6} \Lambda_{4} .
\end{aligned}
$$

For a perfect fluid with equation of state $p=\omega \rho$, the Codazzi equation reads

$$
\dot{\rho}+\frac{\dot{a}}{a}(5 \rho+6 p+\sigma)=0 \Rightarrow \rho=\rho_{0}\left(\frac{a_{0}}{a}\right)^{5+6 \omega}-\epsilon \frac{|\sigma|}{5+6 \omega},
$$




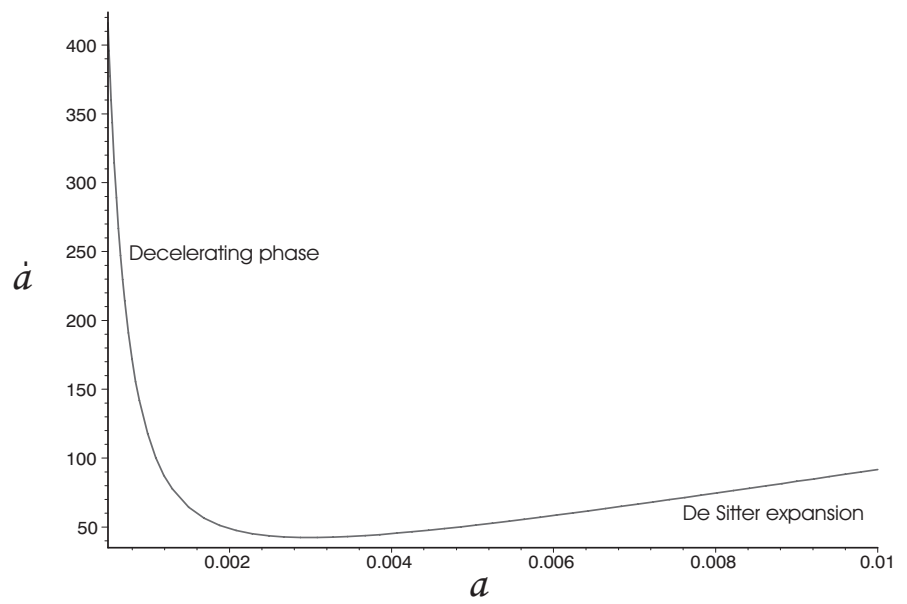

Fig. 1. The transition from a decelerating phase with $\rho \propto a^{-3}$ to a de Sitter expansion with an effective cosmological constant $\rho=\frac{1}{3} \sigma$. Here we consider $\rho_{0}=a_{0}=G_{N}=1$ and $|\sigma|=10^{8}$.

where $\rho_{0}$ and $a_{0}$ can be taken respectively as the value of the energy density and scale factor today. Taking the time derivative of Eq. (37) together with the above Codazzi equation, one re-obtains the dynamical equation (38). This is a consistency check that reassures that our hypothesis of the torsion tensor being continuous across the brane is well defined.

For a timelike extra dimension and $\omega>-5 / 6$, the quadratic term on equation (37) could eventually become relevant in order to avoid the initial singularity. In this case however, the nature of the extra dimension also fixes the sign of the effective four dimensional constant $\left(\Lambda_{4}>0\right)$ and one can show that it is impossible to find bouncing solutions with a timelike extra dimension. In fact, this dynamical system has only one solution that is a static universe with $\rho=\sigma$ and Eq. (39) fixes the value of the scale factor. This static solution is stable in the sense that the constraint equation (37) does not allow the system to move away from the point $\rho=\sigma$. One can also calculate all orders of time derivative of the scale factor and show that they all vanish as they should if the system is constrained to be fix in the static solution $\rho=\sigma$.

In the case of a spacelike extra dimension, $\epsilon=-1$, all the terms on the righthand side of Eq. (37) are now positive definite. Hence, bouncing solutions are not possible. Nevertheless, Eq. (39) shows that in an expanding universe the energy density approaches a positive constant

$$
\lim _{a \rightarrow \infty} \rho \rightarrow \frac{\sigma}{5+6 \omega}
$$

Therefore, if $\omega>-2 / 3$ one can show that the universe starts in a decelerating expanding phase with small scale factor and very high density energy and eventually evolves into an accelerating phase that will tend asymptotically to a de Sitter like 
expansion. Considering $\omega=-1 / 3$ for instance, a spacelike extra dimension can reproduce the transition from a decelerating phase to an accelerating regime with an effective cosmological constant $\rho=\frac{1}{3} \sigma$ (cf. Fig. 1).

\section{Conclusion}

In the present work, we have examined the corrections in the formalism of brane world theory due to the presence of torsion in the affine structure of the bulk manifold for an arbitrary noncompact extra dimension. Considering a five dimensional Einstein field equation in the bulk, we derived the four dimensional modified field equations with extra terms depending on the energy-momentum tensor of the bulk, the extrinsic curvature and torsion components. The hypotheses that these components do not vanish in the bulk induces a torsion tensor in the brane introducing extra correction terms in the field equations.

Assuming the so called $Z_{2}$ symmetry and that the metric tensor is everywhere continuous, we derived the necessary junction conditions which connect the symmetrical part of extrinsic curvature to the matter distribution on the brane. Furthermore, inasmuch as the torsion is an independent tensor and in a sense as fundamental as the metric tensor, we have considered that the torsion is also continuous but its first derivative, which appears in the field equation are discontinuous. The novelty in the junction conditions is related to the anti-symmetric part of the extrinsic curvature given by the 5 -dimension torsion tensor projected into the brane.

The identification of the Newtonian constant, $G_{N}$, fixes the sign of the tension of the brane with respect to the extra dimension. That is, for a timelike extra dimension we have a positive brane tension. On the contrary, for a spacelike extra dimension we have a negative brane tension.

In our cosmological model, the de Sitter (or Anti de Sitter) bulk solution comes from an effective cosmological constant related to torsion terms. Moreover, the cosmological constant in the brane $\Lambda_{4}$ has the same sign of the extra dimension. It is worth to remark that if one assumes a nonvanishing cosmological constant in the five dimensional field equation, then $\Lambda_{4}$ is no longer fixed an in fact does not need to have the same sign as the extra dimension.

Considering that the torsion tensor has only a scalar degree of freedom in our model, we have shown that this ansatz is equivalent to an effective five dimensional cosmological constant, allowing the de Sitter (or Anti de Sitter) solution in the bulk. For a timelike extra dimension, $\epsilon=+1$, there is only a unique solution that describes a static universe. Contrarily to other static solutions in the literature, ${ }^{21-24}$ this solution is stable in the sense that Friedmann's equations do not allow any matter perturbation restricting the scale factor to a fixed value. Despite the presence of an initial singularity for a spacelike extra dimension, $\epsilon=-1$, we have shown how a transition from a decelerating to an accelerating phase can occur. In this case, the universe starts in a decelerating expanding phase with small scale factor and very 
high density energy and eventually evolves into an accelerating phase that will tend asymptotically to a de Sitter like expansion.

\section{Acknowledgements}

We would like to thank CNPq of Brazil for financial support. We would also like to thank 'Pequeno Seminario' of CBPF's Cosmology Group for useful discussions, comments and suggestions.

\section{References}

1. T. Shiromizu, K. Maeda and M. Sasaki, Phys. Rev. D 62, 024012 (2000).

2. L. Randall and R. Sundrum, Phys. Rev. Lett. 83, 4690 (1999).

3. L. Randall and R. Sundrum, Phys. Rev. Lett. 83, 3370 (1999).

4. Y. V. Shtanov, Phys. Lett. B 541, 177 (2002).

5. Y. Shtanov and V. Sahni, Phys. Lett. B 557, 1 (2003).

6. Y. Ma and X. Hang, J. Cosm. Astrop. Phys. 03, 6 (2009).

7. J. H. Brodie and D. A. Easson, J. Cosm. Astrop. Phys. 12, 4 (2003).

8. R. Maier, I. Damião Soares and E. V. Tonini, Phys. Rev. D 79, 023522 (2009).

9. R. T. Hammond, Gen. Rel. Grav. 29, 727 (1997).

10. R. T. Hammond, Gen. Rel. Grav. 42, 2345 (2010).

11. R. T. Hammond, Gen. Rel. Grav. 26, 247 (1994).

12. S. Chandrasekhar, The Mathematical Theory of Black Holes (Oxford University Press, New York, 1983).

13. R. Maier and I. Damião Soares, Int. J. Mod. Phys. D 18, 2221 (2009).

14. R. M. Wald, General Relativity (University of Chicago Press, Chicago, 1984).

15. L. P. Eisenhart, Non-Riemannian Geometry (American Mathematical Society colloquium publications, New York, 1968).

16. W. Israel, Nuovo Cimento B 44, 1 (1966).

17. G. Boillat, J. Math. Phys. 11, 941 (1970).

18. Y. Choquet-Bruhat, C. De Witt-Morette and M. Dillard-Bleick, in Analysis, Manifolds and Physics (North-Holland Publishing, New York, 1977).

19. J. Hadamard, Leçons sur la Propagation des Ondes et les Équations de l'Hydrodynamique (Hermann, Paris, 1903).

20. R. Maier and F. T. Falciano, Phys. Rev. D 83, 064019 (2011).

21. J. D. Barrow, G. Ellis, R. Maartens and C. Tsagas, Class. Quant. Grav. 20, L155 (2003).

22. G. Ellis and R. Maartens, Class. Quant. Grav. 21, 223 (2004).

23. D. J. Mulryne, R. Tavakol, J. E. Lidsey and G. Ellis, Phys. Rev. D 71, 123512 (2005).

24. L. Parisi, M. Bruni, R. Maartens and K. Vandersloot, Class. Quant. Grav. 24, 6243 (2007). 